\title{
ARTICLE
}

Myelodysplastic syndrome

\section{TP53 mutation status divides myelodysplastic syndromes with complex karyotypes into distinct prognostic subgroups}

\author{
Detlef Haase ${ }^{1} \cdot$ Kristen E. Stevenson ${ }^{2}$ - Donna Neuberg $\mathbb{C}^{2}$ - Jaroslaw P. Maciejewski ${ }^{3}$ Aziz Nazha ${ }^{3}$. \\ Mikkael A. Sekeres ${ }^{3}$ - Benjamin L. Ebert ${ }^{2}$ - Guillermo Garcia-Manero ${ }^{4}$ - Claudia Haferlach ${ }^{5} \cdot$ Torsten Haferlach $^{5}$. \\ Wolfgang Kern ${ }^{5}$ - Seishi Ogawa ${ }^{6} \cdot$ Yasunobu Nagata $^{3} \cdot$ Kenichi Yoshida $^{6} \cdot$ Timothy A. Graubert $^{7}$. \\ Matthew J. Walter $\mathbb{1 0}^{8} \cdot$ Alan F. List $^{9} \cdot$ Rami S. Komrokji $^{9}$ - Eric Padron ${ }^{9} \cdot$ David Sallman $^{9}$ - Elli Papaemmanuil ${ }^{10}$. \\ Peter J. Campbell ${ }^{11}$ - Michael R. Savona $\mathbb{D}^{12}$ - Adam Seegmiller ${ }^{12} \cdot$ Lionel Adès $^{13}$ - Pierre Fenaux ${ }^{13}$. \\ Lee-Yung Shih $\mathbb{1}^{14}$. David Bowen ${ }^{15} \cdot$ Michael J. Groves $^{16}$. Sudhir Tauro ${ }^{16}$. Michaela Fontenay ${ }^{17}$. \\ Olivier Kosmider ${ }^{17} \cdot$ Michal Bar-Natan $^{18} \cdot$ David Steensma $^{2} \cdot$ Richard Stone $^{2} \cdot$ Michael Heuser $^{19} \cdot$ Felicitas Thol $^{19}$. \\ Mario Cazzola ${ }^{20} \cdot$ Luca Malcovati $^{20} \cdot$ Aly Karsan $^{21} \cdot$ Christina Ganster $^{1} \cdot$ Eva Hellström-Lindberg $^{22}$. \\ Jacqueline Boultwood ${ }^{23}$ - Andrea Pellagatti $\mathbb{1}^{23}$ - Valeria Santini ${ }^{24} \cdot$ Lynn Quek $^{25,26}$ - Paresh Vyas $\mathbb{1}^{25,26}$. \\ Heinz Tüchler ${ }^{27} \cdot$ Peter L. Greenberg ${ }^{28} \cdot$ Rafael Bejar $\mathbb{1}^{29} \cdot$ for the International Working Group for \\ MDS Molecular Prognostic Committee
}

Received: 28 August 2018 / Revised: 28 November 2018 / Accepted: 5 December 2018 / Published online: 11 January 2019

(c) The Author(s) 2019. This article is published with open access

\begin{abstract}
Risk stratification is critical in the care of patients with myelodysplastic syndromes (MDS). Approximately $10 \%$ have a complex karyotype (CK), defined as more than two cytogenetic abnormalities, which is a highly adverse prognostic marker. However, CK-MDS can carry a wide range of chromosomal abnormalities and somatic mutations. To refine risk stratification of CK-MDS patients, we examined data from 359 CK-MDS patients shared by the International Working Group for MDS. Mutations were underrepresented with the exception of TP53 mutations, identified in 55\% of patients. TP53 mutated patients had even fewer co-mutated genes but were enriched for the $\operatorname{del}(5 q)$ chromosomal abnormality $(p<0.005)$, monosomal karyotype $(p<0.001)$, and high complexity, defined as more than 4 cytogenetic abnormalities $(p<0.001)$. Monosomal karyotype, high complexity, and TP53 mutation were individually associated with shorter overall survival, but monosomal status was not significant in a multivariable model. Multivariable survival modeling identified severe anemia (hemoglobin < 8.0 g/dL), NRAS mutation, SF3B1 mutation, TP53 mutation, elevated blast percentage (>10\%), abnormal 3q, abnormal 9, and monosomy 7 as having the greatest survival risk. The poor risk associated with CK-MDS is driven by its association with prognostically adverse TP53 mutations and can be refined by considering clinical and karyotype features.
\end{abstract}

\section{Introduction}

Risk stratification is essential in the clinical care of patients with myelodysplastic syndromes (MDS). The predicted prognosis helps physicians select when and how to treat and sets expectations for patients and families. Recurrent

Supplementary information The online version of this article (https:// doi.org/10.1038/s41375-018-0351-2) contains supplementary material, which is available to authorized users.

Rafael Bejar

rabejar@ucsd.edu

Extended author information available on the last page of the article. cytogenetic abnormalities are powerful predictors of prognosis in MDS and are included in several prognostic scoring systems used in clinical practice [1]. Individual abnormalities can have a wide range of prognostic associations when present in isolation. For example, deletion of chromosome $5 q$ is favorable while loss of chromosome 7 is adverse [2]. In contrast, the presence of three or more chromosomal abnormalities is always considered adverse, regardless of which lesions are present [3, 4]. Prognostic models such as the Revised International Prognostic Scoring System (IPSSR) assign substantial risk to the $10 \%$ of MDS patients with a complex karyotype (CK), defined as three or more somatic chromosomal abnormalities present in a single clone. The IPSS-R considers patients with exactly three abnormalities to 
have 'Poor' cytogenetic risk, while those with four or more abnormalities have 'Very Poor' cytogenetic risk, the highest possible risk category, with a score that exceeds that assigned to bone marrow blasts $>10 \%[2,5]$. In fact, the presence of CK excludes most MDS patients from having 'lower risk' MDS, as defined by the IPSS-R, in the presence of even one additional risk factor.

While there are no good actors in this traditionally high-risk population, complex karyotype MDS patients represent a heterogeneous group whose overall survival and disease course is affected by factors other than the number of chromosomal abnormalities they carry [3]. The types of abnormalities present, co-occurring somatic mutations, and clinical features all contribute to the actual risk in patients with complex karyotypes. Several groups have examined the prognostic impact of a monosomal karyotype (MK), defined as a complete loss of an autosomal chromosome in the presence of at least one other structural abnormality or additional monosomy, as in practice, most patients with MK also have CK [6]. Parsing complex karyotypes as monosomal can identify MDS patients with even greater risk than predicted by tools like the IPSS-R, although the independent prognostic significance of MK is still debated [7-12]. Other studies have focused on the high frequency of TP53 mutations in patients with complex karyotypes [13-17]. TP53 mutations have highly adverse prognostic implications in a wide variety of clinical settings that are independent of other risk factors [18-25]. This is despite their association with adverse clinical features such as increased blast proportion, severe thrombocytopenia, and multiple chromosomal abnormalities [13-15, 21, 26]. The type and abundance of TP53 mutation in question may further refine its prognostic impact [27-29]. The extent to which TP53 mutations can modify risk assessment in otherwise higher risk MDS patients with multiple chromosomal abnormalities remains unclear.

To examine the impact of somatic mutations in CK-MDS, the International Working Group (IWG) for MDS Molecular Prognosis Committee collected clinical and mutational information about complex karyotype MDS patients evaluated at 19 centers internationally. We examined risk-associated markers in complex karyotype MDS such as the presence of MK, specific chromosomal lesions, total number of lesions, clinical variables, and the presence of TP53 mutations to determine which features had independent prognostic value that could be used to better risk stratify patients with complex karyotype MDS.

\section{Materials and methods}

\section{Patient data collection}

Members of the IWG for MDS shared clinical and mutation data on 359 patients with complex karyotypes collected from 19 centers (Supplemental Table 1) some of whom were included in previously published MDS cohorts [1315, 24, 30]. Patients consented to sample collection, analysis, and clinical annotation at their home institution on protocols approved by local ethics review boards in accordance with the Declaration of Helsinki. All data shared for this study were assigned unique patient identifiers. Anonymized patient data included age, sex, blood counts, bone marrow blast proportion, somatic mutations calls, and conventional G-banded karyotype results. Patients were excluded from further study if they did not meet criteria for a complex karyotype after manual review, had a sequenced sample collected only at the time of stem cell transplantation, or had a diagnosis of acute myeloid leukemia (AML) with $\geq 30 \%$ blasts at the time of sample collection. Patients with oligoblastic AML with up to $29 \%$ blasts were included.

\section{Karyotype review}

Every complex karyotype was manually reviewed and parsed independently by $\mathrm{RB}$ and $\mathrm{DH}$ blinded to the clinical information or TP53 mutation status associated with the patient. Discrepancies in total numbers of chromosome abnormalities, monosomal status, or the presence of specific abnormalities were resolved jointly by RB and DH. A brief schema with examples describing the approach used to count and identify chromosomal abnormalities can be found in Supplemental Table 2.

\section{Mutation assessment}

Each center performed its own sequencing to interrogate the TP53 gene, resulting in a call of presence or absence of a TP53 mutation. This included Sanger sequencing or various forms of next-generation sequencing. Some centers reported only the presence or absence of a TP53 mutation, while others provided the DNA change, the predicted impact on coding amino acid sequence, and the variant allele fraction. Several centers reported the presence or absence of other mutations from larger panels of myeloid malignancyassociated genes.

\section{Statistical analysis}

Patient characteristics were compared between groups using Fisher's exact test for categorical data and the Wilcoxon ranksum test for continuous measures. Overall survival (OS) was measured from the time of sample collection for the determination of mutational status to the time of death from any cause. OS curves were constructed using the method of Kaplan and Meier and compared using the log-rank test. OS was evaluated in Cox proportional hazard regression modeling univariately 
and a stepwise procedure was used to determine a final multivariable model. Patient characteristics (age, sex, bone marrow blast \%, hemoglobin, absolute neutrophil count, and platelet count categorized as shown in the patient characteristic table and IPSS-R), karyotypic features (number of abnormalities, monosomal karyotype, abnormal 17, 17p deletion with predicted loss of the TP53 locus, $-7, \operatorname{del}(7 q), \operatorname{del}(5 q)$, abnormal 3q, der(1;7), abnormal 9, -13/13q, -18/18q, -21, +21 ), and mutational status (including the presence or absence of mutations in TP53, DNMT3A, ASXL1, TET2, U2AF1, RUNX1, JAK2, SF3B1, CBL, NRAS, KRAS, EZH2, SRSF2, $I D H 1$, and $I D H 2$ ) were included as candidates in the modeling where at each step the variable entry criterion was $p<0.20$ and variables were retained in the model if $p<0.05$. Models including IPSS-R did not include its components as candidate variables. A missing indicator was used in modeling for unknown values for a category. A landmark analysis at day 100 post sample collection was used to compare patients who had received a transplant to those who did not. The Welch $t$ test was used to compare the average mutation rate between groups. All tests are reported as two-sided and considered significant at the $<0.05$ level. SAS version 9.4 and RStudio version 0.99.441 with $\mathrm{R}$ version 3.4.1 were used for all analyses.

\section{Results}

\section{Complex karyotype MDS patients have a high frequency of TP53 mutations which are associated with specific clinical features}

Of the 359 MDS patients with CK shared with the IWG for MDS Prognosis Molecular Committee, 339 (94\%) had TP53 sequencing performed. One or more mutations were identified in $186(55 \%)$ cases. Patient characteristics stratified by TP53 mutation status are shown in Table 1 . Of the 186 TP53 mutated patients, 164 (89\%) were evaluable for multiple mutations and $159(85 \%)$ could be analyzed for type of mutation.

As shown in Table 1, TP53 mutations were associated with several prognostically adverse features. This included a lower median platelet count (47 vs. $70 \times 10^{9} / \mathrm{L}, p=0.002$ ) and higher median bone marrow blast percentage (9\% vs. $5 \%, p<$ 0.001 ), both of which are considered unfavorable risk factors in various prognostic scoring systems. No differences in hemoglobin level or absolute neutrophil counts were noted.

\section{TP53 mutations are associated with molecular and cytogenetic abnormalities}

Complex karyotype MDS patients harbor fewer somatic point mutations in genes other than TP53 when compared with non CK-MDS patients [13-15]. The majority of samples in our cohort were tested for somatic mutations in several recurrently mutated MDS genes (Supplemental Table 3). The most frequently mutated genes after TP53 were DNMT3A (31/324, 10\%), ASXL1 (29/319, 9\%), and TET2 (27/318, 8\%), all at rates lower than observed in MDS cohorts unselected by karyotype. Several gene mutations were even more underrepresented in the TP53 mutant patient samples compared to wild-type CK-MDS (Fig. 1a, Supplemental Fig. 1). The TP53 mutant group had fewer mutations of ASXL1 (5\% vs. $15 \%, p=0.003), U 2 A F 1$ ( $3 \%$ vs. $11 \%, \mathrm{p}=0.008)$, and RUNX1 (0.5\% vs. $9 \%, p<0.001)$. A total of 250 patients had 12 core genes sequenced (TP53, ASXL1, RUNX1, U2AF1, DNMT3A, TET2, JAK2, SF3B1, $S R S F 2, N R A S, C B L$, and EZH2). Of the 156 with mutated $T P 53,111(71 \%)$ had no additional gene mutations compared to $47(50 \%)$ of the 94 without a TP53 mutation ( $p=$ 0.001 by Fisher's exact test). The average number of mutated genes in the TP53 mutant group was 0.39 nonTP53 genes/patient, whereas in the TP53 wild-type group, this ratio was $0.81(p<0.001$ by Welch $t$-test).

TP53 mutation status was also associated with the number and types of chromosomal abnormalities present within the complex karyotype. $\operatorname{Del}(5 q)$, monosomy 7 , and abnormalities of chromosome 17 were the most common recurrent cytogenetic findings, present in 156 (43\%), 123 (34\%), and $121(34 \%)$ members of the entire cohort respectively (Fig. 1b, Supplemental Table 4).

Cases with five or more karyotype abnormalities were described as having 'high complexity' (HC) (Fig. 2a) given the marked difference in OS at this cut point (Fig. 3c). HC was found in $86 \%$ of TP53 mutant patients compared with $53 \%$ of those without an identified TP53 mutation $(p<$ 0.001). TP53 mutation status was also associated with MK, a feature that has frequently been cited as an independent prognostic measure in MDS and AML [7, 8, 11, 12, 31-34]. Eighty-eight percent of the TP53 mutant patients had MK compared to $61 \%$ without the mutation $(p<0.001)$. These distinct methods of describing the complex karyotype, $\mathrm{HC}$ and $\mathrm{MK}$, demonstrate significant overlap and association with TP53 mutation status as $42 \%$ of patients harbored all three features (Fig. 2b).

\section{Karyotype abnormalities and TP53 mutation are associated with OS}

As a group, this cohort with CK-MDS patients had a poor outcome, with a median OS of only 0.9 years (Fig. 3a). Even shorter OS might be expected in the TP53 mutant subset given the associations between TP53 mutation status and the adverse clinical and cytogenetic measures described above. Indeed, CK-MDS patients with TP53 mutation had a significantly greater hazard ratio (HR) of 
Table 1 Patient demographics and laboratory values

\begin{tabular}{|c|c|c|c|c|}
\hline & $N(\%)$ & TP53 $\mathrm{WT}^{\mathrm{a}}$ & TP53 mut $^{\mathrm{a}}$ & $P$ value ${ }^{\mathrm{b}}$ \\
\hline$N$ & 359 & 153 & 186 & \\
\hline Age, median (range) & $68(23,94)$ & $67(34,89)$ & $70(23,94)$ & 0.096 \\
\hline$<50$ Years & $28(8)$ & $12(8)$ & $15(8)$ & 0.22 \\
\hline 50-59 Years & $55(15)$ & $25(16)$ & $25(13)$ & \\
\hline 60-69 Years & $107(30)$ & $51(33)$ & $49(26)$ & \\
\hline $70-80$ Years & $135(37)$ & $56(37)$ & $73(39)$ & \\
\hline$\geq 80$ Years & $33(10)$ & $9(6)$ & $23(12)$ & \\
\hline Unknown & $1(<1)$ & $0(0)$ & $1(<1)$ & \\
\hline \multicolumn{5}{|l|}{ Sex } \\
\hline Male & $223(62)$ & $102(67)$ & $107(58)$ & 0.093 \\
\hline Female & $136(38)$ & $51(33)$ & $79(42)$ & \\
\hline Bone marrow blast $\%$, median (range) & $7(0,28)$ & $5(0,27)$ & $9(0,28)$ & $<0.001$ \\
\hline$<5 \%$ & $135(38)$ & $69(45)$ & $54(29)$ & 0.001 \\
\hline $5-10 \%$ & $104(29)$ & $39(25)$ & $59(32)$ & \\
\hline $11-20 \%$ & $101(28)$ & $35(23)$ & $65(35)$ & \\
\hline $21-29 \%$ & $6(2)$ & $2(1)$ & $3(2)$ & \\
\hline Unknown & $13(4)$ & $8(5)$ & $5(3)$ & \\
\hline \multicolumn{5}{|l|}{ IPSS-R risk group } \\
\hline Very low & $0(0)$ & $0(0)$ & $0(0)$ & $<0.001$ \\
\hline Low & $5(1)$ & $4(3)$ & $1(<1)$ & \\
\hline Intermediate & $26(7)$ & $15(10)$ & $6(3)$ & \\
\hline High & $73(20)$ & $39(25)$ & $29(16)$ & \\
\hline Very high & $224(62)$ & $78(51)$ & $136(73)$ & \\
\hline Unknown & $31(9)$ & $17(11)$ & $14(8)$ & \\
\hline Hemoglobin, median (range) & $9.4(3.7,17.0)$ & $9.4(3.7,17.0)$ & $9.2(5.3,13.5)$ & 0.43 \\
\hline$<8.0(\mathrm{~g} / \mathrm{dL})$ & $61(17)$ & $29(19)$ & $30(16)$ & 0.85 \\
\hline $8.0-9.99(\mathrm{~g} / \mathrm{dL})$ & $161(45)$ & $67(44)$ & $85(46)$ & \\
\hline $10.0-11.99(\mathrm{~g} / \mathrm{dL})$ & $102(28)$ & $40(26)$ & $55(30)$ & \\
\hline$\geq 12.0(\mathrm{~g} / \mathrm{dL})$ & $23(6)$ & $14(9)$ & $7(4)$ & \\
\hline Unknown & $12(3)$ & $3(2)$ & $9(5)$ & \\
\hline Absolute neutrophil count (ANC), median (range) & $1.10(0,35.0)$ & $1.31(0,17.27)$ & $0.94(0,35.0)$ & 0.22 \\
\hline$<0.5\left(\times 10^{3} / \mu \mathrm{L}\right)$ & $62(17)$ & $28(18)$ & $32(17)$ & 0.49 \\
\hline $0.5-1.8\left(\times 10^{3} / \mu \mathrm{L}\right)$ & $145(40)$ & $62(41)$ & $74(40)$ & \\
\hline $1.8-9.99\left(\times 10^{3} / \mu \mathrm{L}\right)$ & $101(28)$ & $45(29)$ & $47(25)$ & \\
\hline$\geq 10\left(\times 10^{3} / \mu \mathrm{L}\right)$ & $7(2)$ & $5(3)$ & $2(1)$ & \\
\hline Unknown & $44(12)$ & $13(8)$ & $31(17)$ & \\
\hline Platelet count, median (range) & $58(4,1073)$ & $70(5,1073)$ & $47(5,693)$ & 0.002 \\
\hline$<50\left(\times 10^{3} / \mu \mathrm{L}\right)$ & $152(42)$ & $50(33)$ & $93(50)$ & $<0.001$ \\
\hline $50-99\left(\times 10^{3} / \mu \mathrm{L}\right)$ & $89(25)$ & $40(26)$ & $46(25)$ & \\
\hline $100-149\left(\times 10^{3} / \mu \mathrm{L}\right)$ & 49 (14) & $24(16)$ & $22(12)$ & \\
\hline $150-449\left(\times 10^{3} / \mu \mathrm{L}\right)$ & $46(13)$ & $26(17)$ & $15(8)$ & \\
\hline$\geq 450\left(\times 10^{3} / \mu \mathrm{L}\right)$ & $5(1)$ & $4(3)$ & $1(1)$ & \\
\hline Unknown & $18(5)$ & $9(6)$ & $9(5)$ & \\
\hline
\end{tabular}

${ }^{\mathrm{a}}$ TP53 mutation status was unknown for 20 samples

${ }^{\mathrm{b}}$ Test excludes unknown categories 
A

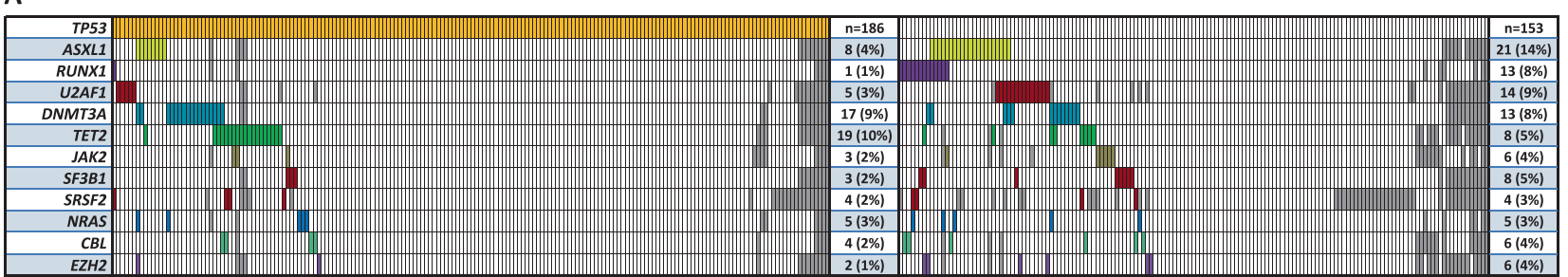

B

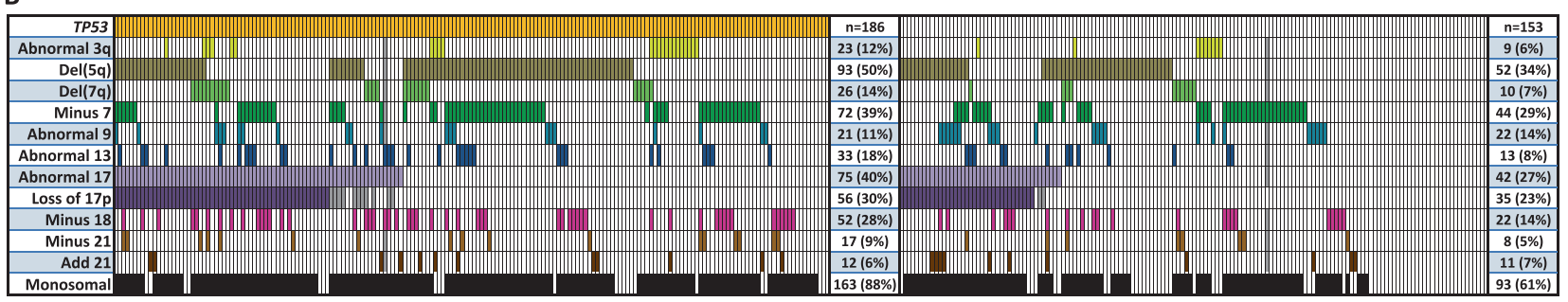

Fig. 1 Select somatically mutated genes and karyotype abnormalities. a Co-mutation plot for somatically mutated genes in complex karyotype MDS patients with and without mutated TP53 (left and right panels, respectively). Each column represents an individual patient. A colored bar indicates a mutation of the gene in that row with gray bars indicating missing data. The last column indicates the number of patients with a mutation of each gene. b Plot of recurrent karyotype

abnormalities in patients with and without mutated TP53 (left and right panels, respectively) using the same schema as in (a). TP53 mutant patients had a higher rate of del(5q) abnormality (50\% vs. $34 \%, p=$ $0.004)$, abnormal chromosome 13 (18\% vs. $8 \%, p=0.017)$, abnormal chromosome 17 (40\% vs. 27\%, $p=0.016$ ), abnormal chromosome 18 ( $28 \%$ vs. $14 \%, p=0.004)$, and $\operatorname{del}(7 \mathrm{q})(14 \%$ vs. $7 \%, p=0.033)$, but a lower rate of $\operatorname{der}(1 ; 7)(\mathrm{q} 10 ; \mathrm{p} 10)(<1 \%$ vs. $5 \%, p=0.025)$

A

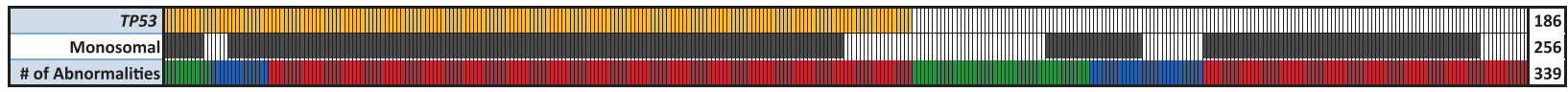

B

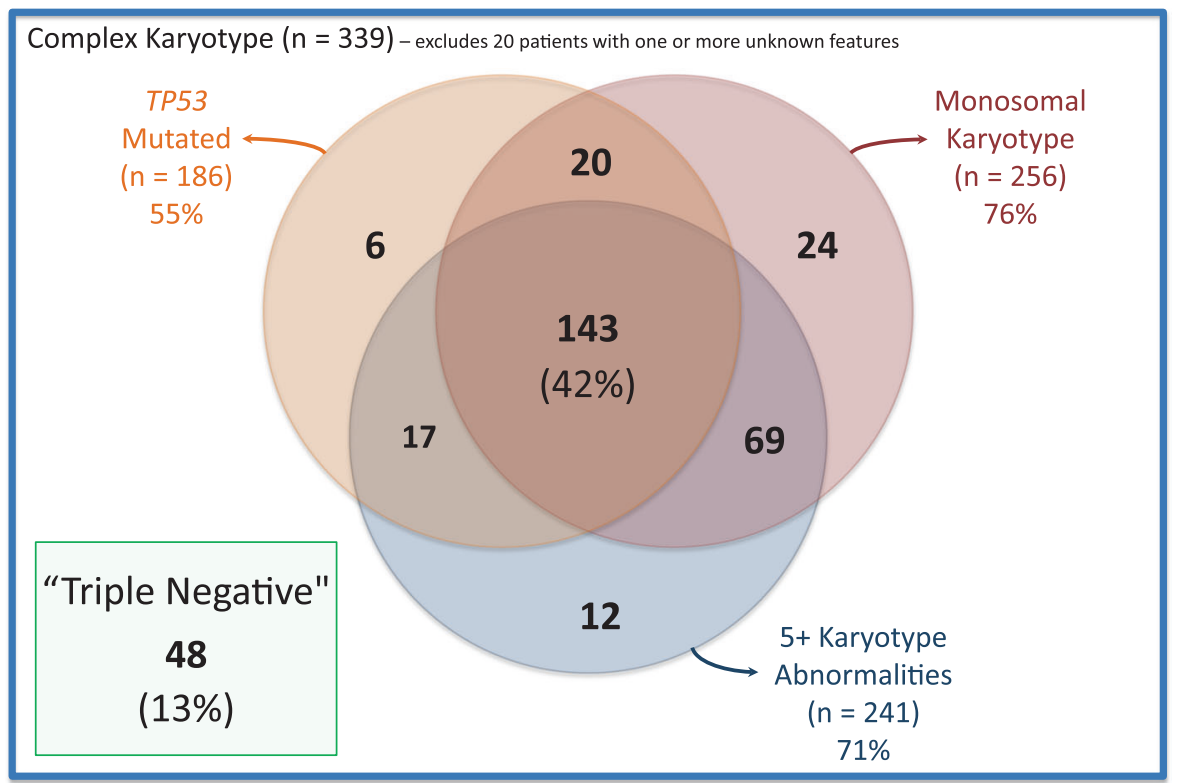

Fig. 2 Interaction between TP53 mutation, monosomy, and number of karyotype abnormalities. a Each column represents an individual patient with orange and black bars indicating TP53 mutation and monosomal karyotype respectively. Colored bars in the last row indicate the number of karyotype abnormalities with green representing 3 , blue representing 4 , and red representing 5 or more. b Venn diagram showing number of cases with overlapping features 


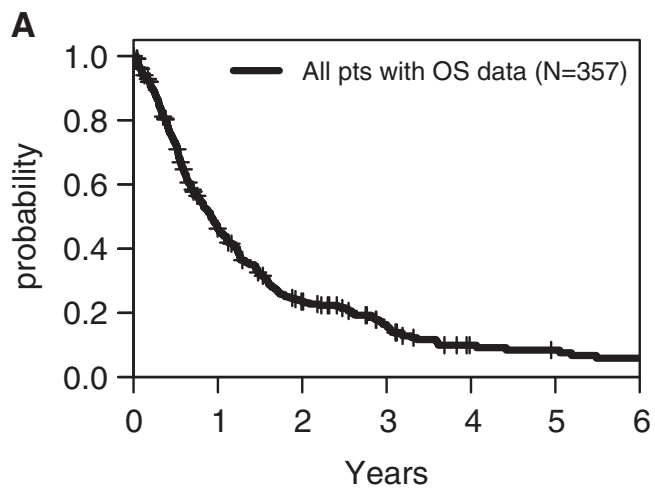

C

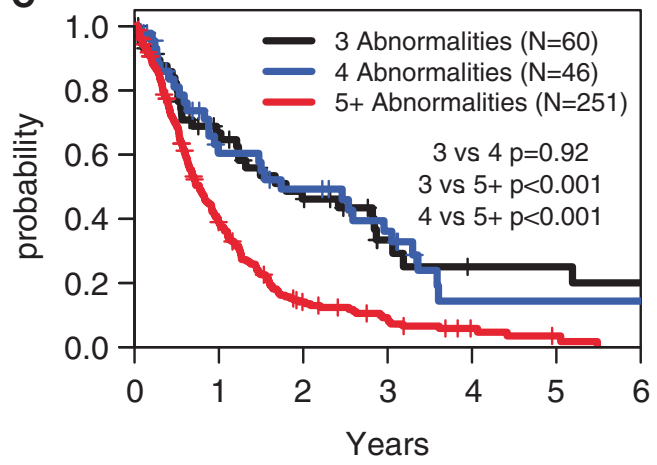

E

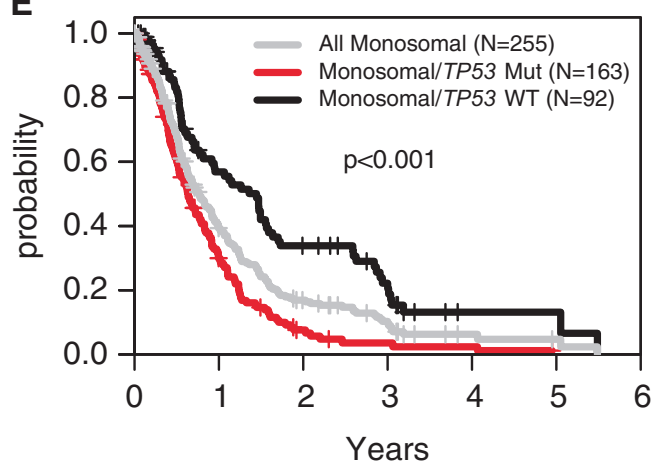

Fig. 3 Overall survival by TP53 mutation, high complexity, and monosomal karyotype status. a Overall survival of the entire cohort. b Overall survival stratified by TP53 mutation status. c Overall survival stratified by the number of clonal karyotype abnormalities. d Overall

death $(2.57 ; 95 \%$ confidence interval (CI) $1.97-3.34, p<$ 0.001 ) with a median OS of 0.6 years compared to 1.5 years for TP53 wild-type patients (Fig. 3b). No other gene mutation was significantly associated with OS in univariate analyses.

Prior studies of MDS patients unselected by karyotype have demonstrated that the prognostic significance of TP53 mutations depends in part on their variant allele frequency (VAF), with smaller clones having a less adverse impact $[27,29]$. To determine whether this holds true in complex karyotype MDS, we examined the survival of 151 patients with TP53 mutations and available VAF data. Nearly two-
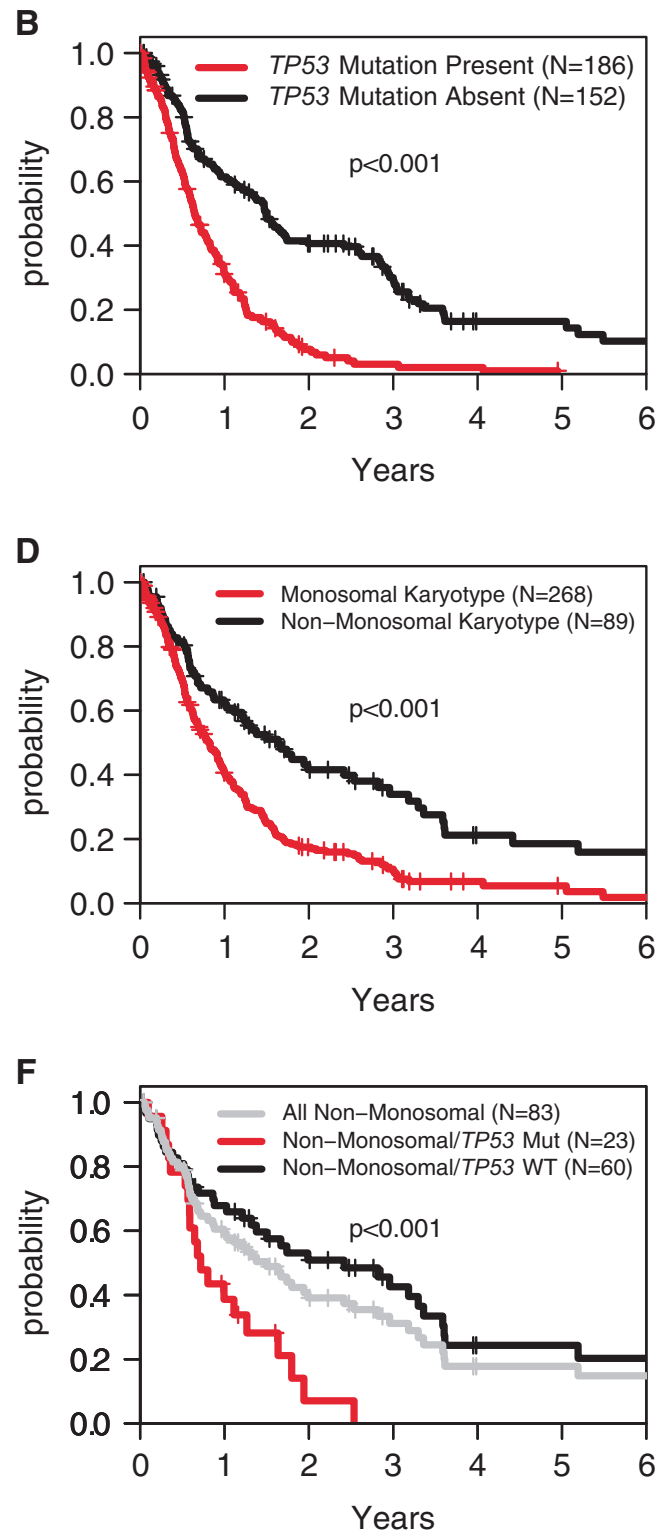

survival stratified by monosomal karyotype status. e Stratification of overall survival by TP53 mutation status in patients with a monosomal karyotype. f Stratification of overall survival by TP53 mutation status in patients without a monosomal karyotype

thirds of TP53 mutant patients had a VAF $>0.4$, with a significantly shorter median OS than those with a VAF $\leq 0.4$ (0.6 vs. 1.1 years, $p=0.004$; Supplemental Fig. 2A). However, mutated patients with a TP53 VAF $\leq 0.4$ still had an inferior survival compared with TP53 wild-type patients (1.1 vs. 1.5 years, $p=0.001$ ). While TP53 VAF was not adjusted for copy number in this analysis, the results were similar in the subset of patients without loss of $17 p$ in their karyotype ( $p=0.014$ for TP53 VAF $\leq 0.4$ vs. $>0.40$; Supplemental Fig. 2B).

The number and type of mutations in TP53 had less impact on OS. Less than $15 \%$ of the cohort carried more 
Table 2 Overall survival modeling of TP53 mutation and karyotype features

\begin{tabular}{|c|c|c|c|c|}
\hline \multirow{2}{*}{$\begin{array}{l}\text { Overall survival model } \\
\text { Considered features }\end{array}$} & \multicolumn{2}{|l|}{ Univariable } & \multicolumn{2}{|l|}{ Multivariable } \\
\hline & $\operatorname{HR}[95 \% \mathrm{CI}]$ & $P$ value & $\operatorname{HR}[95 \% \mathrm{CI}]$ & $P$ value \\
\hline Monosomal yes vs. no & $1.95[1.46-2.62]$ & $<0.001$ & $1.26[0.91-1.75]$ & 0.17 \\
\hline Number of abnormalities $\geq 5$ vs. 4 or 3 & $2.26[1.70-3.02]$ & $<0.001$ & $1.61[1.16-2.24]$ & 0.004 \\
\hline TP53 mutation vs. no mutation & $2.57[1.97-3.34]$ & $<0.001$ & $2.12[1.61-2.79]$ & $<0.001$ \\
\hline Unknown vs. no mutation & $0.70[0.38-1.31]$ & 0.27 & 0.69 [0.37-1.29] & 0.25 \\
\hline
\end{tabular}

than one TP53 mutation, and this was not associated with any difference in survival compared to those harboring only 1 mutation $(p=0.77)$. In contrast, an increase in median OS was noted for missense mutations $(n=126)$ compared with potentially more disruptive types of mutations (frameshift, nonsense, and splice site; $n=33$ ) among the 159 patients with mutation-type data available ( $p=0.016$; Supplemental Fig. 3). Complete loss of the TP53 locus through deletion of chromosome $17 \mathrm{p}$ is not routinely captured by gene sequencing, but could have the same effect as a TP53 mutation. However, cytogenetic abnormalities predicted to cause copy number loss at the TP53 locus had no prognostic impact regardless of TP53 mutations status (Supplemental Fig. 4), suggesting that loss of a TP53 allele by cytogenetic analysis might not be biologically equivalent to a TP53 point mutation in CK-MDS [2, 5, 35, 36]. Further testing of this hypothesis would require examination with more reliable methods including TP53-specific fluorescence in situ hybridization (FISH) probes or copy number-sensitive genomic arrays.

To determine how HC or MK could impact prognosis, we examined OS in patients stratified by these measures. Individually, MK and having five or more karyotype abnormalities were associated with inferior OS (Fig. 3c, d). However, in a multivariable model that considered TP53 mutation, $\mathrm{MK}$, and $\mathrm{HC}$, the presence of $\mathrm{MK}$ was no longer statistically significant (Table 2). Indeed, TP53 mutation status could strongly stratify survival of patients with and without MK (Fig. 3e, f). Double negative patients, defined as having neither TP53 mutation nor HC, had markedly better outcomes with a median OS of 2.6 years compared with 0.6 years $(p<0.001)$ for TP53 mutant and 1.2 years $(p$ $<0.001$ ) for TP53 wild type but with HC (Fig. 4, Supplemental Figure 5).

\section{Multivariable prognostic modeling of OS}

While the two-component model above can risk stratify CK-MDS patients, it does not consider the potential contributions of individual karyotype abnormalities, other gene mutations, or clinical measures that have significant univariate associations with OS (Supplemental Table 5). To explore the prognostic value of these features, we performed multivariable stepwise Cox regression modeling of OS in our cohort.

Candidate variables included age, sex, blood counts, bone marrow blast percentage, mutations in sequenced genes, and the presence of the specific karyotype abnormalities listed in Supplemental Table 5. TP53 mutation was the most significant genetic risk factor, with a HR of 2.67 (Table 3) followed by mutations of SF3B1 and NRAS (Supplemental Figure 6). Cytogenetic features in the final model included monosomy 7 and abnormalities of chromosomes $3 q$ and 9 . These factors had the greatest impact in patients without a TP53 mutation, although monosomy 7 was associated with a shorter OS even in the TP53 mutant group (Supplemental Figure 7). The only clinical factors to retain independent prognostic significance were elevated bone marrow blast percentage and low hemoglobin concentration (Supplemental Figure 8). Importantly, consideration of sample origin (univariate $p=0.18$ ) during model building did not alter the significance of other covariates and was not retained (data not shown). Repeating the multivariable analysis with IPSS-R risk groups in place of bone marrow blast percentage and blood counts as candidate variables gave similar results with IPSS-R high (HR 3.27 ) and very high (HR 4.54) risk groups retained in the final model (Supplemental Table 6; Supplemental Figure 9). Most of the prior model variables remained significant with monosomy 21 as the only additional risk factor observed. In both models, TP53 mutation status remained the most frequently occurring risk factor not currently considered by existing prognostic scoring systems.

\section{Discussion}

Complex karyotype MDS includes a diverse collection of patients typically labeled as having a very poor prognosis $[2,4,5]$. Here we examined data from 359 CK-MDS patients evaluated at multiple centers around the world to determine which factors might improve current risk stratification methods. Collectively, these patients shared features that distinguished them from MDS patients without complex karyotypes. In addition to greater structural genomic instability and a high frequency of TP53 mutations 


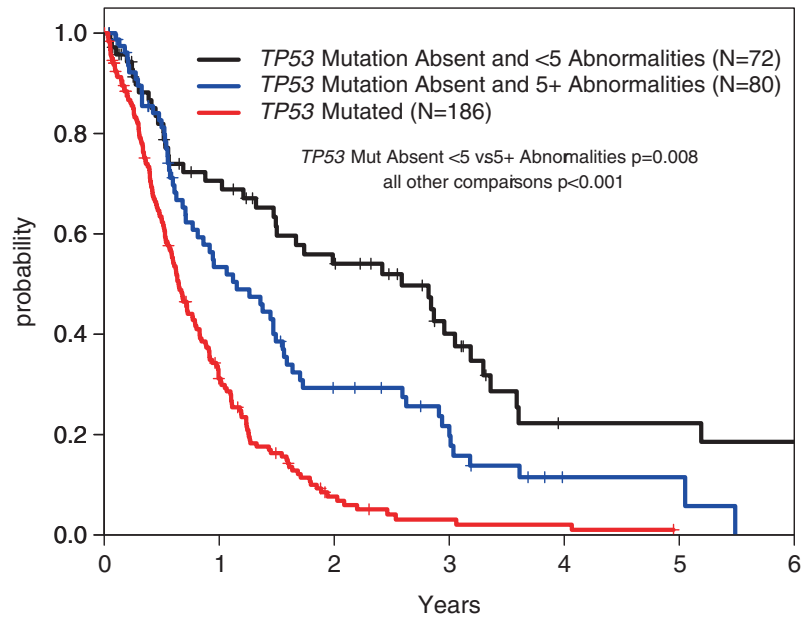

Fig. 4 Overall survival stratified by TP53 mutation and high complexity status

(55\%), patients with CK-MDS had fewer somatic mutations in other MDS-associated genes. These differences were even more pronounced in the TP53 mutant subset of CKMDS, which were more likely to have high complexity, monosomal karyotypes, certain chromosomal abnormalities, and an even lower number of co-mutated myeloid malignancy genes. TP53 mutant CK-MDS patients also had significantly higher bone marrow blast proportion and lower platelet counts, two factors strongly associated with elevated prognostic risk considered by clinical scoring systems like the IPSS-R. Indeed, TP53 mutant CK-MDS patients had an OS that was less than half of that for non-mutant CK-MDS. This powerful adverse prognostic association was statistically independent of other risk factors, including having a higher number of karyotype abnormalities, which together overrode the prognostic impact of the monosomal karyotype.

The consideration of monosomal karyotype as a more accurate risk factor than karyotype complexity in MDS and AML has been controversial [9]. First, not all studies agree on the effect of MK on survival $[6,7,10]$. Second, the vast majority of studies examining the prognostic impact of MK in MDS did not evaluate TP53 mutation status or HC, missing these potential confounders strongly associated with MK [8, 12, 37]. Finally, the definition of MK is not recognized by the International System for Human Cytogenetic Nomenclature (ISCN) and can be problematic to identify in practice $[38,39]$. Some cases of apparent monosomies may be due to complicated unbalanced rearrangements and not truly representative of loss of a complete chromosome. Short of performing 24-color metaphase FISH, this can be difficult to measure reliably. Our results suggest that specific monosomies can retain prognostic significance after consideration of HC and TP53 mutation status, but the more problematic designation of MK does not. Assessment of just HC and TP53 mutation status constitutes a relatively simple means of identifying the roughly $20 \%$ of CK-MDS patients predicted to have an OS that resembles that of IPSS-R intermediate risk patients.

Consideration of multiple clinical, cytogenetic, and molecular features identifies TP53 mutation among the most significant prognostic factor in patients with CK-MDS, yet it remains the only marker not routinely assessed in clinical practice. Here we demonstrate that the presence of TP53 mutation has an independent impact on prognosis that is as great as having severe anemia and greater than having a bone marrow blast proportion of $10-29 \%$. The muted impact of increased blast proportion and the absence of severe thrombocytopenia as independent risk factors are likely due to the association of these features with TP53 mutations. The impact of a TP53 mutation is pronounced even in patients assigned to the very high risk group by the IPSS-R (Supplemental Figure 9). Mutations of SF3B1 and $N R A S$, while rare, were also associated with a greater HR of death. NRAS mutations are known to be adverse in a variety of contexts [40, 41], but SF3B1 mutations are typically considered favorable in MDS [14, 15, 42, 43]. In the context of a complex karyotype, SF3B1 mutations appear adverse, much like in rare cases of SF3B1-mutated AML [44]. Factors that might explain this association were not evident in our small subset of 11 SF3B1 mutant cases. Future prognostic scoring systems that include molecular features will need to consider the interaction between somatic mutations and more traditional risk factors. In the meantime, patients with CK-MDS considered to have a poor prognosis with tools like the IPSS-R can be further risk stratified by consideration of the features in our survival model.

The value of identifying TP53 mutations in MDS may extend beyond their prognostic significance. This study and others have demonstrated that TP53 mutant MDS patients share clinical and genetic features that distinguish them from patients with wild-type TP53. In addition to a higher bone marrow blast proportion, lower platelet count, and greater likelihood of having a high number of chromosomal aberrations, TP53 mutant patients relapse quickly after various forms of treatment [20, 22, 24, 45, 46]. Hematopoietic clones defined by TP53 mutations are enriched after chemotherapy exposure and in therapy-related MDS, suggesting they harbor intrinsic resistance to genotoxic stress [47-50]. TP53 mutations may also help select therapy. For example, novel agents, like APR-246 that specifically target missense mutations of TP53 are in development [51]. As a consequence, TP53 mutant CK-MDS could be considered a distinct subtype of disease with common genetic, clinical, and therapy-related features.

Potential limitations of this multi-center, retrospective analysis include possible differences in patient features and clinical practice patterns as well as the variety of sequencing 
Table 3 Cox regression modeling of overall survival

\begin{tabular}{|c|c|c|c|c|c|}
\hline & $\begin{array}{l}\text { Univariable } \\
\text { HR }[95 \% \mathrm{CI}]\end{array}$ & $P$ value & $\begin{array}{l}\text { Final multivariable } \\
\text { HR }[95 \% \mathrm{CI}]\end{array}$ & $P$ value & $\begin{array}{l}N(\%) \\
\text { non-reference group }\end{array}$ \\
\hline \multicolumn{6}{|l|}{ Final model $^{\mathrm{a}}$} \\
\hline \multicolumn{6}{|l|}{ Gene mutations } \\
\hline TP53 mutation vs. no mutation & $2.56[1.96-3.33]$ & $<0.001$ & $2.67[2.01-3.53]$ & $<0.001$ & $185(52)$ \\
\hline Unknown vs. no mutation & $0.70[0.38-1.31]$ & 0.27 & $1.24[0.46-3.36]$ & 0.68 & $19(5)$ \\
\hline SF3B1 mutation vs. no mutation ${ }^{\mathrm{b}}$ & $1.26[0.62-2.56]$ & 0.52 & $2.81[1.34-5.89]$ & 0.006 & $11(3)$ \\
\hline Unknown vs. no mutation & $1.24[0.46-3.36]$ & 0.68 & $0.75[0.42-1.35]$ & 0.34 & $33(9)$ \\
\hline$N R A S$ mutation vs. no mutation ${ }^{\text {b }}$ & $1.79[0.88-3.63]$ & 0.11 & $2.50[1.21-5.16]$ & 0.013 & $10(3)$ \\
\hline Unknown vs. no mutation & $0.61[0.38-0.98]$ & 0.043 & $0.89[0.38-2.10]$ & 0.79 & $33(9)$ \\
\hline \multicolumn{6}{|l|}{ Cytogenetic abnormalities } \\
\hline-7 Yes vs. no & $1.80[1.40-2.31]$ & $<0.001$ & $1.66[1.28-2.17]$ & $<0.001$ & $120(34)$ \\
\hline Abnormal 3q yes vs. no & $1.99[1.33-2.98]$ & $<0.001$ & $1.85[1.23-2.79]$ & 0.003 & $33(9)$ \\
\hline Abnormal 9 yes vs. no & $1.47[1.02-2.11]$ & 0.037 & $1.90[1.31-2.77]$ & $<0.001$ & $45(13)$ \\
\hline \multicolumn{6}{|l|}{ Clinical features } \\
\hline \multicolumn{6}{|l|}{ Blast $\%$} \\
\hline $5-10 \%$ vs. $<5 \%$ & $1.41[1.03-1.91]$ & 0.030 & $1.24[0.90-1.71]$ & 0.20 & $104(29)$ \\
\hline $11-30 \%$ vs. $<5 \%$ & $2.05[1.53-2.75]$ & $<0.001$ & 1.68 [1.24-2.29] & $<0.001$ & $106(29)$ \\
\hline Unknown vs. $<5 \%$ & $1.12[0.60-2.09]$ & 0.73 & $1.20[0.63-2.30]$ & 0.58 & $12(3)$ \\
\hline \multicolumn{6}{|l|}{ Hemoglobin (g/dL) } \\
\hline $10.0-11.99$ vs. $\geq 12.0$ & $1.97[1.09-3.58]$ & 0.025 & $1.30[0.71-2.38]$ & 0.40 & $102(29)$ \\
\hline $8.0-9.99$ vs. $\geq 12.0$ & $2.71[1.53-4.81]$ & $<0.001$ & $1.72[0.96-3.11]$ & 0.071 & $160(45)$ \\
\hline$<8.0$ vs. $\geq 12.0$ & $3.67[1.97-6.86]$ & $<0.001$ & $2.93[1.53-5.62]$ & 0.001 & $58(16)$ \\
\hline Unknown vs. $\geq 12.0$ & 2.60 [1.11-6.09] & 0.028 & $1.52[0.64-3.62]$ & 0.35 & $12(3)$ \\
\hline
\end{tabular}

${ }^{a}$ Modeling performed for 355 patients, excluding 2 patients with unknown survival status and 2 with incomplete karyotype information

${ }^{\mathrm{b}}$ Of the 11 patients with SF3B1 mutations, 3 also had TP53 mutation, and of the 10 patients with NRAS mutations, 5 had a TP53 mutation

methods and analysis pipelines at each institution. Not all centers reported the type, number, or VAFs of TP53 mutations identified and data on time to AML transformation was not available. However, sample origin was not a significant confounder in our multivariable analyses. Information about treatment status was incomplete or absent in over a third of the cohort, although no disease-modifying therapy, including stem cell transplantation, has been definitively shown to mitigate the adverse impact of TP53 mutation. Only 27 patients (8\%) were reported as having received a stem cell transplant and the transplant status was not known for the majority of patients. Similarly, whether patients had primary vs. therapy-related MDS (t-MDS) was not known for 86 patients (24\%). Only 21 patients (6\%) were reported as having t-MDS. These measures had little impact on OS (Supplementary Figure 10).

\section{Conclusion}

This study has several important strengths. It examines a large cohort of CK-MDS patients powered to find strong associations between clinical and genetic disease features including OS. It validates and expands upon results from many prior smaller studies. This consistency and the multiinstitutional nature of the cohort imply that our conclusions are robust and generalizable. Finally, our findings support modifications to the standard of care for CK-MDS patients to include routine genetic sequencing of TP53. These mutations modify risk assessment even in CK-MDS patients traditionally considered to have the greatest disease risk. TP53 mutation status is the most significant risk marker in this population missing from prognostic tools used in clinical practice. Cytogenetics alone appears insufficient for the evaluation of CK-MDS patients and routine testing for TP53 mutations should be considered in this population.

Acknowledgements We would like to thank the MDS Foundation for their support of the International Working Group for MDS and Celgene for funding the efforts of the IWG-prognosis molecular subcommittee and this study. RB was additionally supported by K08 DK091360. PV acknowledges funding from the MRC Disease Team Awards (G1000729/94931 and MR/L008963/1) MRC Molecular Haematology Unit and the Oxford Partnership Comprehensive Biomedical Research Centre (NIHR BRC Funding scheme. oxfbrc2012-1). LQ is funded by an MRC Clinician Scientist Fellowship. $\mathrm{JB}$ and $\mathrm{AP}$ acknowledge funding from Bloodwise (UK). LM 
acknowledges funding from Associazione Italiana per la Ricerca sul Cancro (AIRC, Individual Grant 20125). MH acknowledges funding from DFG grant HE 5240/6-1. In addition, we would like to thank the following for their help with this study: Jin Shao from Washington University in St. Louis, USA; Professor Mathilde Hunault-Berger from CHU Angers, France; Professor Norbert Vey from Institut Paoli Calmettes, Marseille, France; Michael Byrne, P Brent Ferrell, David R Head, Ridas Juskevicius, Carrie Kitko, Emily F Mason, Sanjay R Mohan, Claudio A Mosse, Tamara K Moyo, Aaron C Shaver, Andrew L Sochacki, and Stephen A Strickland from Vanderbilt University.

Author contributions Patient data were contributed by member institutions of the IWG listed in Supplemental Table 1. RB and DH manually reviewed and parsed karyotype information. KS and DN assembled the patient data and performed all of the statistical analyses. $\mathrm{RB}, \mathrm{DH}, \mathrm{KS}$, and DN wrote the manuscript which was further revised by EP, PN, MAS, and the IWG Manuscript Committee and reviewed by all coauthors.

\section{Compliance with ethical standards}

Conflict of interest RB has served as a consultant for Genoptix and Celgene and served on advisory boards for Otsuka/Astex, AbbVie/ Genetech, and Celgene and has received research funding from Celgene and Takeda. DH has served as consultant and advisory board member for Celgene and Novartis from both of which he has received research funding. PV receives research funding from Celgene and has been on advisory boards for Celgene, Pfizer, Novartis, Jazz, Daiichi Sanko. LQ receives research funding from Celgene. MAS has served on an advisory board for Celgene. MRE reports consultancy and research funding from Astex, Incyte, Karyopharm, Sunesis, Takeda, and TG Therapeutics; equity in Karyopharm; and DSMB membership for Celgene and Gilead. TH, CH, and WK report partial ownership of MLL-Munich Leukemia Laboratory. All other authors declare that they have no conflict of interest.

Publisher's note: Springer Nature remains neutral with regard to jurisdictional claims in published maps and institutional affiliations.

Open Access This article is licensed under a Creative Commons Attribution 4.0 International License, which permits use, sharing, adaptation, distribution and reproduction in any medium or format, as long as you give appropriate credit to the original author(s) and the source, provide a link to the Creative Commons license, and indicate if changes were made. The images or other third party material in this article are included in the article's Creative Commons license, unless indicated otherwise in a credit line to the material. If material is not included in the article's Creative Commons license and your intended use is not permitted by statutory regulation or exceeds the permitted use, you will need to obtain permission directly from the copyright holder. To view a copy of this license, visit http://creativecommons. org/licenses/by/4.0/.

\section{References}

1. Bejar R. Clinical and genetic predictors of prognosis in myelodysplastic syndromes. Haematologica. 2014;99:956-64.

2. Schanz J, Tuchler H, Sole F, Mallo M, Luno E, Cervera J, et al. New comprehensive cytogenetic scoring system for primary myelodysplastic syndromes (MDS) and oligoblastic acute myeloid leukemia after MDS derived from an international database merge. J Clin Oncol. 2012;30:820-9.
3. Schanz J, Steidl C, Fonatsch C, Pfeilstocker M, Nosslinger T, Tuechler $\mathrm{H}$, et al. Coalesced multicentric analysis of 2,351 patients with myelodysplastic syndromes indicates an underestimation of poor-risk cytogenetics of myelodysplastic syndromes in the international prognostic scoring system. J Clin Oncol. 2011;29:1963-70.

4. Greenberg P, Cox C, LeBeau MM, Fenaux P, Morel P, Sanz G, et al. International scoring system for evaluating prognosis in myelodysplastic syndromes. Blood. 1997;89:2079-88.

5. Greenberg PL, Tuechler H, Schanz J, Sanz G, Garcia-Manero G, Sole F, et al. Revised international prognostic scoring system for myelodysplastic syndromes. Blood. 2012;120:2454-65.

6. Anelli L, Pasciolla C, Zagaria A, Specchia G, Albano F. Monosomal karyotype in myeloid neoplasias: a literature review. Onco Targets Ther. 2017;10:2163-71.

7. Patnaik MM, Hanson CA, Hodnefield JM, Knudson R, Van Dyke DL, Tefferi A. Monosomal karyotype in myelodysplastic syndromes, with or without monosomy 7 or 5 , is prognostically worse than an otherwise complex karyotype. Leukemia. 2011;25:26670.

8. Cluzeau T, Mounier N, Karsenti JM, Richez V, Legros L, Gastaud $\mathrm{L}$, et al. Monosomal karyotype improves IPSS-R stratification in MDS and AML patients treated with Azacitidine. Am J Hematol. 2013;88:780-3.

9. Schanz J, Tuchler H, Sole F, Mallo M, Luno E, Cervera J, et al. Monosomal karyotype in MDS: explaining the poor prognosis? Leukemia. 2013;27:1988-95.

10. Valcarcel D, Adema V, Sole F, Ortega M, Nomdedeu B, Sanz G, et al. Complex, not monosomal, karyotype is the cytogenetic marker of poorest prognosis in patients with primary myelodysplastic syndrome. J Clin Oncol. 2013;31:916-22.

11. Yang YT, Hou HA, Liu CY, Lin CC, Chou WC, Lee FY, et al. IPSS-R in 555 Taiwanese patients with primary MDS: Integration of monosomal karyotype can better risk-stratify the patients. Am J Hematol. 2014;89:19.

12. McQuilten ZK, Sundararajan V, Andrianopoulos N, Curtis DJ, Wood EM, Campbell LJ, et al. Monosomal karyotype predicts inferior survival independently of a complex karyotype in patients with myelodysplastic syndromes. Cancer. 2015;121:2892-9.

13. Bejar R, Stevenson K, Abdel-Wahab O, Galili N, Nilsson B, Garcia-Manero $\mathrm{G}$, et al. Clinical effect of point mutations in myelodysplastic syndromes. N Engl J Med. 2011;364:2496-506.

14. Papaemmanuil E, Gerstung M, Malcovati L, Tauro S, Gundem G, Van Loo P, et al. Clinical and biological implications of driver mutations in myelodysplastic syndromes. Blood. 2013;122:361627. quiz 99

15. Haferlach T, Nagata Y, Grossmann V, Okuno Y, Bacher U, Nagae $\mathrm{G}$, et al. Landscape of genetic lesions in 944 patients with myelodysplastic syndromes. Leukemia. 2014;28:241-7.

16. Haferlach C, Dicker F, Herholz H, Schnittger S, Kern W, Haferlach T. Mutations of the TP53 gene in acute myeloid leukemia are strongly associated with a complex aberrant karyotype. Leukemia. 2008;22:1539-41.

17. Bally C, Ades L, Renneville A, Sebert M, Eclache V, Preudhomme C, et al. Prognostic value of TP53 gene mutations in myelodysplastic syndromes and acute myeloid leukemia treated with azacitidine. Leuk Res. 2014;38:751-5.

18. Welch JS, Petti AA, Miller CA, Fronick CC, O'Laughlin M, Fulton RS, et al. TP53 and decitabine in acute myeloid leukemia and myelodysplastic syndromes. N Engl J Med. 2016;375:202336.

19. Della Porta MG, Galli A, Bacigalupo A, Zibellini S, Bernardi M, Rizzo E, et al. Clinical effects of driver somatic mutations on the outcomes of patients with myelodysplastic syndromes treated with allogeneic hematopoietic stem-cell transplantation. J Clin Oncol. 2016;34:3627-37. 
20. Bejar R, Stevenson KE, Caughey B, Lindsley RC, Mar BG, Stojanov $\mathrm{P}$, et al. Somatic mutations predict poor outcome in patients with myelodysplastic syndrome after hematopoietic stemcell transplantation. J Clin Oncol. 2014;32:2691-8.

21. Lindsley RC, Saber W, Mar BG, Redd R, Wang T, Haagenson $\mathrm{MD}$, et al. Prognostic mutations in myelodysplastic syndrome after stem-cell transplantation. N Engl J Med. 2017;376:536-47.

22. Montalban-Bravo G, Takahashi K, Patel K, Wang F, Xingzhi S, Nogueras GM, et al. Impact of the number of mutations in survival and response outcomes to hypomethylating agents in patients with myelodysplastic syndromes or myelodysplastic/ myeloproliferative neoplasms. Oncotarget. 2018;9:9714-27.

23. Craddock CF, Houlton AE, Quek LS, Ferguson P, Gbandi E, Roberts $\mathrm{C}$, et al. Outcome of azacitidine therapy in acute myeloid leukemia is not improved by concurrent vorinostat therapy but is predicted by a diagnostic molecular signature. Clin Cancer Res. 2017;23:6430-40.

24. Bejar R, Lord A, Stevenson K, Bar-Natan M, Perez-Ladaga A, Zaneveld $\mathrm{J}$, et al. TET2 mutations predict response to hypomethylating agents in myelodysplastic syndrome patients. Blood. 2014;124:2705-12.

25. Yoshizato T, Nannya Y, Atsuta Y, Shiozawa Y, Iijima-Yamashita $\mathrm{Y}$, Yoshida $\mathrm{K}$, et al. Genetic abnormalities in myelodysplasia and secondary acute myeloid leukemia: impact on outcome of stem cell transplantation. Blood. 2017;129:2347-58.

26. Kulasekararaj AG, Smith AE, Mian SA, Mohamedali AM, Krishnamurthy P, Lea NC, et al. TP53 mutations in myelodysplastic syndrome are strongly correlated with aberrations of chromosome 5, and correlate with adverse prognosis. Br J Haematol. 2013;160:660-72.

27. Sallman DA, Komrokji R, Vaupel C, Cluzeau T, Geyer SM, McGraw KL, et al. Impact of TP53 mutation variant allele frequency on phenotype and outcomes in myelodysplastic syndromes. Leukemia. 2016;30:666-73.

28. Goel S, Hall J, Pradhan K, Hirsch C, Przychodzen B, Shastri A, et al. High prevalence and allele burden-independent prognostic importance of p53 mutations in an inner-city MDS/AML cohort. Leukemia. 2016;30:1793-5.

29. Wang W, Routbort MJ, Tang Z, Ok CY, Patel KP, Daver N, et al. Characterization of TP53 mutations in low-grade myelodysplastic syndromes and myelodysplastic syndromes with a non-complex karyotype. Eur J Haematol. 2017;99:536-43.

30. Malcovati L, Galli A, Travaglino E, Ambaglio I, Rizzo E, Molteni $\mathrm{E}$, et al. Clinical significance of somatic mutation in unexplained blood cytopenia. Blood. 2017;129:3371-8.

31. Breems DA, Van Putten WL, De Greef GE, Van Zelderen-Bhola SL, Gerssen-Schoorl KB, Mellink CH, et al. Monosomal karyotype in acute myeloid leukemia: a better indicator of poor prognosis than a complex karyotype. J Clin Oncol. 2008;26:4791-7.

32. Kayser S, Zucknick M, Dohner K, Krauter J, Kohne CH, Horst HA, et al. Monosomal karyotype in adult acute myeloid leukemia: prognostic impact and outcome after different treatment strategies. Blood. 2012;119:551-8.

33. Koenecke C, Gohring G, de Wreede LC, van Biezen A, Scheid C, Volin L, et al. Impact of the revised International Prognostic Scoring System, cytogenetics and monosomal karyotype on outcome after allogeneic stem cell transplantation for myelodysplastic syndromes and secondary acute myeloid leukemia evolving from myelodysplastic syndromes: a retrospective multicenter study of the European Society of Blood and Marrow Transplantation. Haematologica. 2015;100:400-8.

34. Wudhikarn K, Van Rheeden R, Leopold C, Rattanaumpawan P, Gingrich R, de Magalhaes Silverman M. Outcome of allogeneic stem cell transplantation in myelodysplastic syndrome patients: prognostic implication of monosomal karyotype. Eur J Haematol. 2012;89:294-301.
35. Stengel A, Kern W, Haferlach T, Meggendorfer M, Fasan A, Haferlach C. The impact of TP53 mutations and TP53 deletions on survival varies between AML, ALL, MDS and CLL: an analysis of 3307 cases. Leukemia. 2017;31:705-11.

36. Sebaa A, Ades L, Baran-Marzack F, Mozziconacci MJ, Penther D, Dobbelstein $\mathrm{S}$, et al. Incidence of $17 \mathrm{p}$ deletions and TP53 mutation in myelodysplastic syndrome and acute myeloid leukemia with 5q deletion. Genes Chromosomes Cancer. 2012; 51:1086-92.

37. Itzykson R, Thepot S, Eclache V, Quesnel B, Dreyfus F, BeyneRauzy $\mathrm{O}$, et al. Prognostic significance of monosomal karyotype in higher risk myelodysplastic syndrome treated with azacitidine. Leukemia. 2011;25:1207-9.

38. McGowan-Jordan J, Simons A, Schmid M (eds). An International System for Human Cytogenomic Nomenclature (2016). Cytogenetic and Genome Research. Basel, Switzerland 2016;149.

39. Galvan AB, Mallo M, Arenillas L, Salido M, Espinet B, Pedro C, et al. Does monosomy 5 really exist in myelodysplastic syndromes and acute myeloid leukemia? Leuk Res. 2010;34:1242-5.

40. Murphy DM, Bejar R, Stevenson K, Neuberg D, Shi Y, Cubrich $\mathrm{C}$, et al. NRAS mutations with low allele burden have independent prognostic significance for patients with lower risk myelodysplastic syndromes. Leukemia. 2013;27:2077-81.

41. Takahashi K, Jabbour E, Wang X, Luthra R, Bueso-Ramos C, Patel K, et al. Dynamic acquisition of FLT3 or RAS alterations drive a subset of patients with lower risk MDS to secondary AML. Leukemia. 2013;27:2081-3.

42. Malcovati L, Karimi M, Papaemmanuil E, Ambaglio I, Jadersten M, Jansson M, et al. SF3B1 mutation identifies a distinct subset of myelodysplastic syndrome with ring sideroblasts. Blood. 2015;126:233-41.

43. Malcovati L, Papaemmanuil E, Ambaglio I, Elena C, Galli A, Della Porta MG, et al. Driver somatic mutations identify distinct disease entities within myeloid neoplasms with myelodysplasia. Blood. 2014;124:1513-21.

44. Papaemmanuil E, Gerstung M, Bullinger L, Gaidzik VI, Paschka P, Roberts ND, et al. Genomic classification and prognosis in acute myeloid leukemia. N Engl J Med. 2016; 374:2209-21.

45. Jadersten M, Saft L, Smith A, Kulasekararaj A, Pomplun S, Gohring G, et al. TP53 mutations in low-risk myelodysplastic syndromes with del $(5 q)$ predict disease progression. J Clin Oncol. 2011;29:1971-9.

46. Scharenberg C, Giai V, Pellagatti A, Saft L, Dimitriou M, Jansson $\mathrm{M}$, et al. Progression in patients with low- and intermediate-1-risk $\operatorname{del}(5 \mathrm{q})$ myelodysplastic syndromes is predicted by a limited subset of mutations. Haematologica. 2017;102:498-508.

47. Lindsley RC, Mar BG, Mazzola E, Grauman PV, Shareef S, Allen $\mathrm{SL}$, et al. Acute myeloid leukemia ontogeny is defined by distinct somatic mutations. Blood. 2015;125:1367-76.

48. Wong TN, Miller CA, Jotte MRM, Bagegni N, Baty JD, Schmidt $\mathrm{AP}$, et al. Cellular stressors contribute to the expansion of hematopoietic clones of varying leukemic potential. Nat Commun. 2018;9:455.

49. Gibson CJ, Lindsley RC, Tchekmedyian V, Shi J, Mar BG, Jaiswal S, et al. Clonal hematopoiesis associated with adverse outcomes following autologous stem cell transplantation for nonHodgkin lymphoma. Blood. 2016;128:986.

50. Coombs CC, Zehir A, Devlin SM, Kishtagari A, Syed A, Jonsson $\mathrm{P}$, et al. Therapy-related clonal hematopoiesis in patients with non-hematologic cancers is common and associated with adverse clinical outcomes. Cell Stem Cell. 2017;21:374-82 e4.

51. Deneberg S, Cherif H, Lazarevic V, Andersson PO, von Euler M, Juliusson G, et al. An open-label phase I dose-finding study of APR-246 in hematological malignancies. Blood Cancer J. 2016;6:e447. 


\section{Affiliations}

\section{Detlef Haase $^{1} \cdot$ Kristen E. Stevenson $^{2}$ - Donna Neuberg $\mathbb{D}^{2}$ - Jaroslaw P. Maciejewski ${ }^{3}$ - Aziz Nazha ${ }^{3}$.} Mikkael A. Sekeres ${ }^{3}$ - Benjamin L. Ebert ${ }^{2} \cdot$ Guillermo Garcia-Manero $^{4}$. Claudia Haferlach ${ }^{5} \cdot$ Torsten Haferlach $^{5}$. Wolfgang Kern ${ }^{5}$ - Seishi Ogawa ${ }^{6} \cdot$ Yasunobu Nagata ${ }^{3} \cdot$ Kenichi Yoshida $^{6} \cdot$ Timothy A. Graubert $^{7}$. Matthew J. Walter $\mathbb{1}^{8} \cdot$ Alan F. List $^{9} \cdot$ Rami S. Komrokji $^{9}$ - Eric Padron ${ }^{9} \cdot$ David Sallman $^{9}$ - Elli Papaemmanuil ${ }^{10}$. Peter J. Campbell ${ }^{11}$ - Michael R. Savona $\mathbb{1 D}^{12}$ - Adam Seegmiller ${ }^{12} \cdot$ Lionel Adès $^{13}$ • Pierre Fenaux ${ }^{13}$. Lee-Yung Shih $\mathbb{1}^{14} \cdot$ David Bowen $^{15} \cdot$ Michael J. Groves $^{16} \cdot$ Sudhir Tauro $^{16} \cdot$ Michaela Fontenay $^{17}$. Olivier Kosmider $^{17} \cdot$ Michal Bar-Natan ${ }^{18} \cdot$ David Steensma $^{2} \cdot$ Richard Stone $^{2} \cdot$ Michael Heuser $^{19} \cdot$ Felicitas Thol $^{19}$. Mario Cazzola ${ }^{20}$ - Luca Malcovati ${ }^{20}$ - Aly Karsan $^{21}$. Christina Ganster ${ }^{1}$ - Eva Hellström-Lindberg ${ }^{22}$. Jacqueline Boultwood ${ }^{23}$ - Andrea Pellagatti $\mathbb{1}^{23} \cdot$ Valeria Santini ${ }^{24} \cdot$ Lynn Quek $^{25,26}$ - Paresh Vyas $\mathbb{( D}^{25,26}$. Heinz Tüchler ${ }^{27} \cdot$ Peter L. Greenberg ${ }^{28} \cdot$ Rafael Bejar $\mathbb{1}^{29}$ for the International Working Group for MDS Molecular Prognostic Committee

1 University Medical Center, Georg- August-University, Goettingen, Germany

2 Dana-Farber Cancer Institute, Boston, MA, USA

3 Cleveland Clinic Taussig Cancer Center, Cleveland, OH, USA

4 University of Texas MD Anderson Cancer Center, Houston, TX, USA

5 MLL Munich Leukemia Laboratory, Munich, Germany

6 Kyoto University, Kyoto, Japan

7 Massachusetts General Hospital Cancer Center, Boston, MA, USA

8 Washington University School of Medicine, St. Louis, MO, USA

9 H. Lee Moffitt Cancer Center and Research Institute, Tampa Bay, FL, USA

10 Memorial Sloan Kettering Cancer Center, New York, NY, USA

11 Wellcome Trust Sanger Institute, Cambridge, UK

12 Vanderbilt-Ingram Cancer Center, Nashville, TN, USA

13 Hôpital St Louis, Assistance Publique-Hôpitaux de Paris and Paris Diderot University, Paris, France

14 Chang Gung Memorial Hospital and Chang Gung University, Taoyuan, Taiwan

15 St. James's Institute of Oncology, Leeds Teaching Hospitals, Leeds, UK

16 University of Dundee, Ninewells Hospital, Dundee, UK
17 Université Paris Descartes, Hopital Cochin Assistance PubliqueHopitaux de Paris, Paris, France

18 Tisch Cancer Institute, Icahn School of Medicine at Mount Sinai, New York, NY, USA

19 Hannover Medical School, Hannover, Germany

20 Fondazione IRCCS Policlinico San Matteo \& University of Pavia, Pavia, Italy

21 University of British Columbia, Vancouver, BC, Canada

22 Karolinska Institutet, Karolinska University Hospital, Stockholm, Sweden

23 Radcliffe Department of Medicine, University of Oxford, Oxford, UK

24 MDS Unit, AOU Careggi, University of Florence, Florence, Italy

25 MRC Molecular Hematology Unit, WIMM University of Oxford, Oxford, UK

26 Haematology Theme Oxford Biomedical Research Centre and Department of Hematology, Oxford University Hospitals NHS Foundation Trust, Oxford, UK

27 Ludwig-Boltzmann Institute for Leukemia Research, Vienna, Austria

28 Stanford University Cancer Institute, Stanford, CA, USA

29 UC San Diego Moores Cancer Center, La Jolla, CA, USA 